\title{
Retropupillary iris claw intraocular lens implantation in penetrating keratoplasty patients.
}

\author{
Reshma Raj ${ }^{*}$, Vasanthi Kotian, Vijay Pai \\ Department of K.S. Hegde Charitable Hospital, Nithyanandanagar, Derlaketta, Mangalore, India.
}

\begin{abstract}
Objective-To evaluate the visual outcome and assess the complication rate after implantation of a retropupillary iris-claw Intraocular Lens (IOL) during Penetrating Keratoplasty (PKP).

Method-A descriptive study on 15 eyes of 15 patients underwent penetrating keratoplasty along with retropupillary iris claw intraocular lens implantation. Reasons for penetrating keratoplasty included pseudophakic bullous keratopathy, aphakic bullous keratopathy, and leukomatous corneal opacity. Mean follow up was 3 months for Visual Acuity (VA), Intraocular Pressure (IOP), graft clarity and any complications.

RESULT-Of 15 eyes, visual acuity improvement was seen in $11(74 \%)$ eyes and remained unchanged in $4(26 \%)$ eyes. Post operatively elevated IOP was seen in $8(53 \%)$ eyes, which was managed medically. Improvement in graft clarity was noted in 7 (46\%) eyes 1 week following the surgery.

Conclusion-The results demonstrate that penetrating keratoplasty combined with retropupillary Iris claw lens is an easy and effective method for the correction of aphakia in patients with no capsule support.
\end{abstract}

Keywords: Penetrating keratoplasty, Intraocular lens, Aphakia, Keratopathy.

Accepted on October 03, 2018

\section{Introduction}

The iris claw IOL attached to the anterior iris was developed by Worst in 1972 [1]. He has provided a technique to correct aphakia in the absence of capsular support and without direct angle compromise. However, significant complication is damage to endothelium [2], particularly in patients with narrow anterior chambers and in corneal transplantation. The technique was modifies by Brasse and Neuhann [3] by clipping the lens to the posterior iris, thereby protecting the endothelium, with the A contant altered accordingly to 117.0 [4].

Intraocular lens implantation in eyes with pseudophakic or aphakic corneal edema and insufficient posterior capsular support is a surgical challenge [4]. The iris claw lens is fixated to the iris without sutures which is a faster procedure when compared to scleral fixating lens in combined penetrating keratoplasty $[4,5]$. The iris claw lens has the advantage that it can be fixated to the iris without sutures because the peripheral iris is incarcerated between the claws. Since an implantation of iris claw lenses only takes a few minutes, the hypotonic open sky phase can be shortened compared to combined surgery with the scleral fixation technique in cases of combined penetrating keratoplasty $[5,6]$.

The purpose of this study is to evaluate the visual outcome and assess the complication rate after implantation of a retropupillary iris-claw Intraocular Lens (IOL) during Penetrating Keratoplasty (PKP).

\section{Method}

This study was conducted during July 2016 to July 2017 at K. S. Hegde Hospital, Mangalore. Our study included 15 eyes of 15 patients and all patients were operated by a single surgeon. Patients included were pseudophakic bullous keratopathy due to Anterior Chamber Intraocular Lens (ACIOL), aphakic bullous keratopathy, leucomatous corneal opacity with lack of posterior capsular support.

Patients underwent the following combined procedures:

1. Penetrating keratoplasty.

2. Anterior vitrectomy-if required.

3. Release of PAS- if needed.

4. PMMA iris claw lens fixation before graft suturing.

All patients underwent corneal trephination with trephine. After making side ports, the recipient corneal button was cut out with scissors (Figure 1). Removal of angle supported IOL implanted previously (Figure 2) was followed by anterior vitrectomy for few cases. The retropupillary iris claw lens was then enclaved with convex side down by an open sky approach on the mid peripheral iris. The donor's corneal button was then sutured to recipient bed with 10-0 nylon sutures. Post-operatively, patients were examined on the 1 st, $7^{\text {th }}$, and $30^{\text {th }}$ post-operative days, and then 3 months in the follow-up period (Figure 3). Best Corrected Visual Acuity (BCVA) and Intraocular Pressure (IOP) were noted and compared to preoperative data. The graft clarity was assessed by slit-lamp examination. 


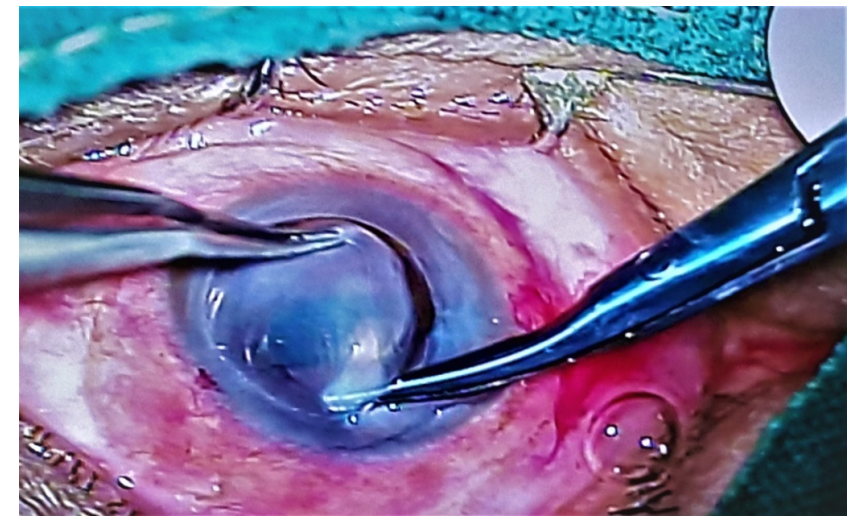

Figure 1: Recipient corneal button cut with scissors.

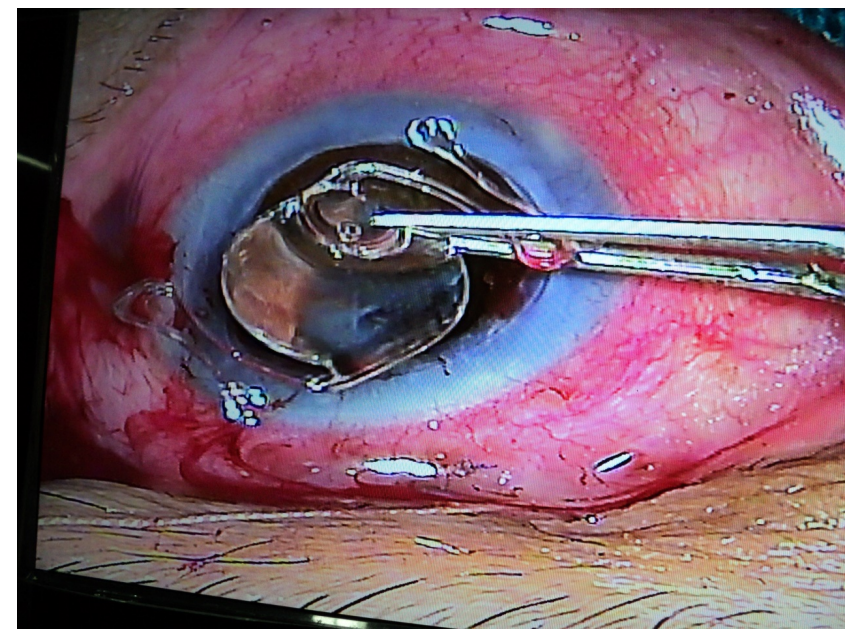

Figure 2: Removal of $A C I O L$.

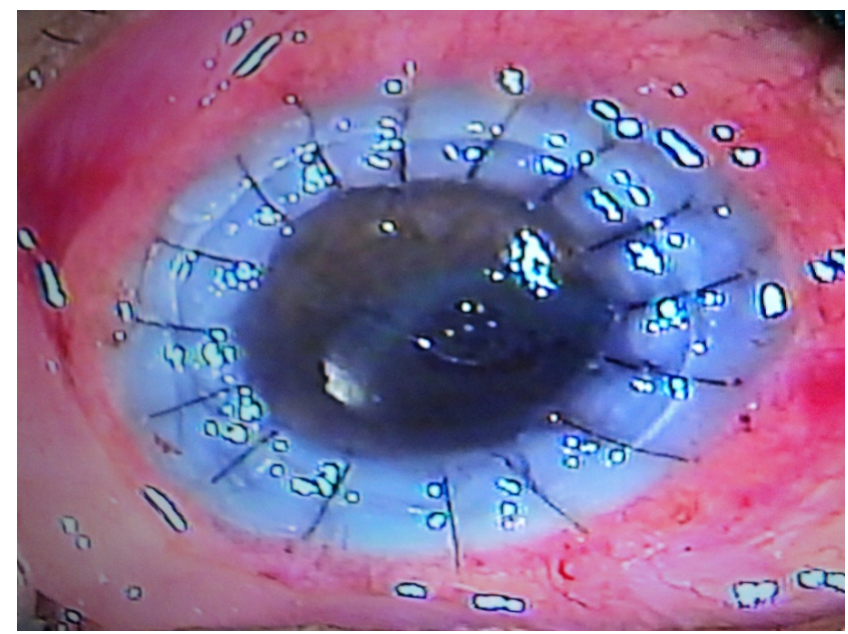

Figure 3: Post op PKP with iris claw implantation.

\section{Study design}

This is a cross-sectional, non-interventional, descriptive and hospital based study. The collected information is summarized by using frequency and percentage.

\section{Statistical analysis}

The results were statistically analysed. Statistical analysis was performed on SPSS version 17.0 for Windows. Descriptive statistics such as frequency and percentage for qualitative data and mean with standard deviation for quantitative data were used.

\section{Results}

The mean age of patients was 55.3 (range of 45 to $65 \mathrm{yrs}$.) $80 \%$ of patients were males and $20 \%$ females. Of 15 patients, 8 were diagnosed with pseudophakic bullous keratopathy, 4 with aphakic bullous keratopathy and 3 with leukomatous corneal opacity. Pre-op Visual acuity (Figure 4) in our study ranged from perception of light to counting finger $1 \mathrm{~m}$. Post-op visual acuity (Figure 5) ranged from perception of light to 6/18.

\section{Preop BCVA}

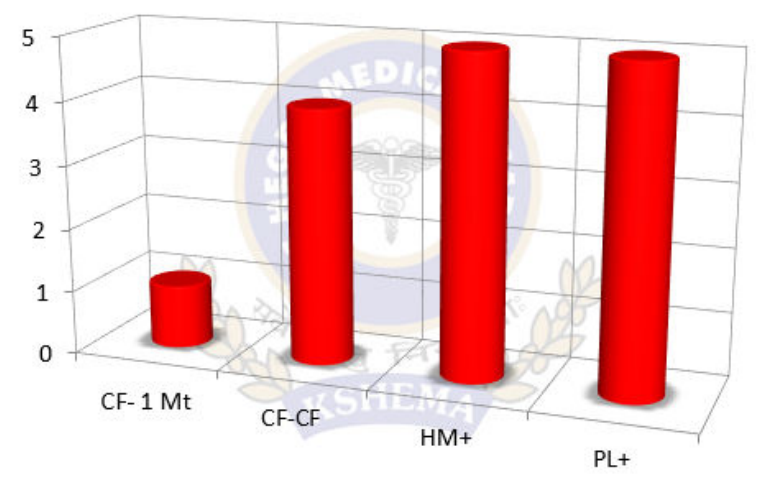

Figure 4: Pre-operative visual acuity of patients ranged from Counting Finger (CF) $1 \mathrm{~m}$ to Perception of Light (PL).

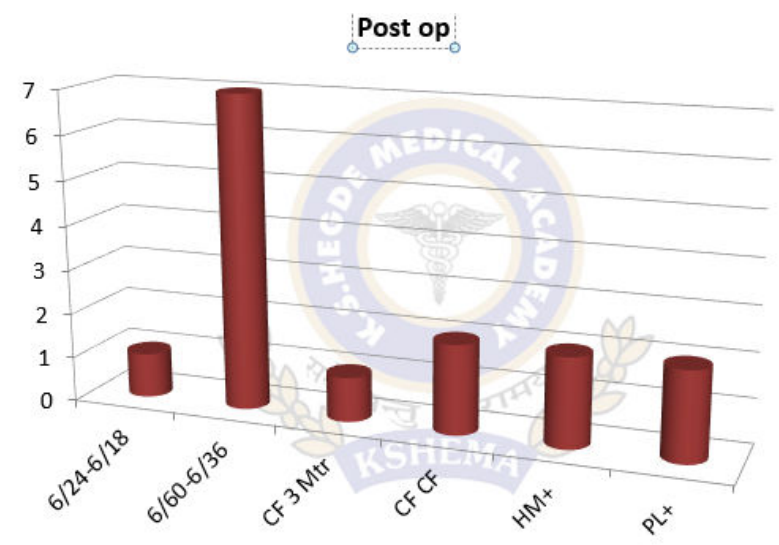

Figure 5: Post-op visual acuity of patients after 3 months ranged from 6/18 (Snellen's chart) to Perception of Light (PL).

Regarding IOP, 75\% of aphakic bullous keratopathy had raised IOP, when compared with $50 \%$ of PBK and $30 \%$ of leucomatous corneal opacities (Table 1).

Table 1: Intraocular pressure range.

\begin{tabular}{|l|l|l|l|}
\hline IOP & PBK & ABK & LEUCO OPACITY \\
\hline$<21 \mathrm{mmHg}$ & 4 & 1 & 2 \\
\hline
\end{tabular}


Citation: Raj R, Kotian V, Pai V. Retropupillary iris claw intraocular lens implantation in penetrating keratoplasty patients. J Clin Ophthalmol 2018;2(2):92-5.

\begin{tabular}{|l|l|l|l|}
\hline $22-30 \mathrm{mmHg}$ & 4 & 3 & 1 \\
\hline
\end{tabular}

Graft clarity (Figure $6 \&$ Table 2) [7] was also assessed at the end of 3 months. In this $46.6 \%$ of patients had grade 4 graft clarity.

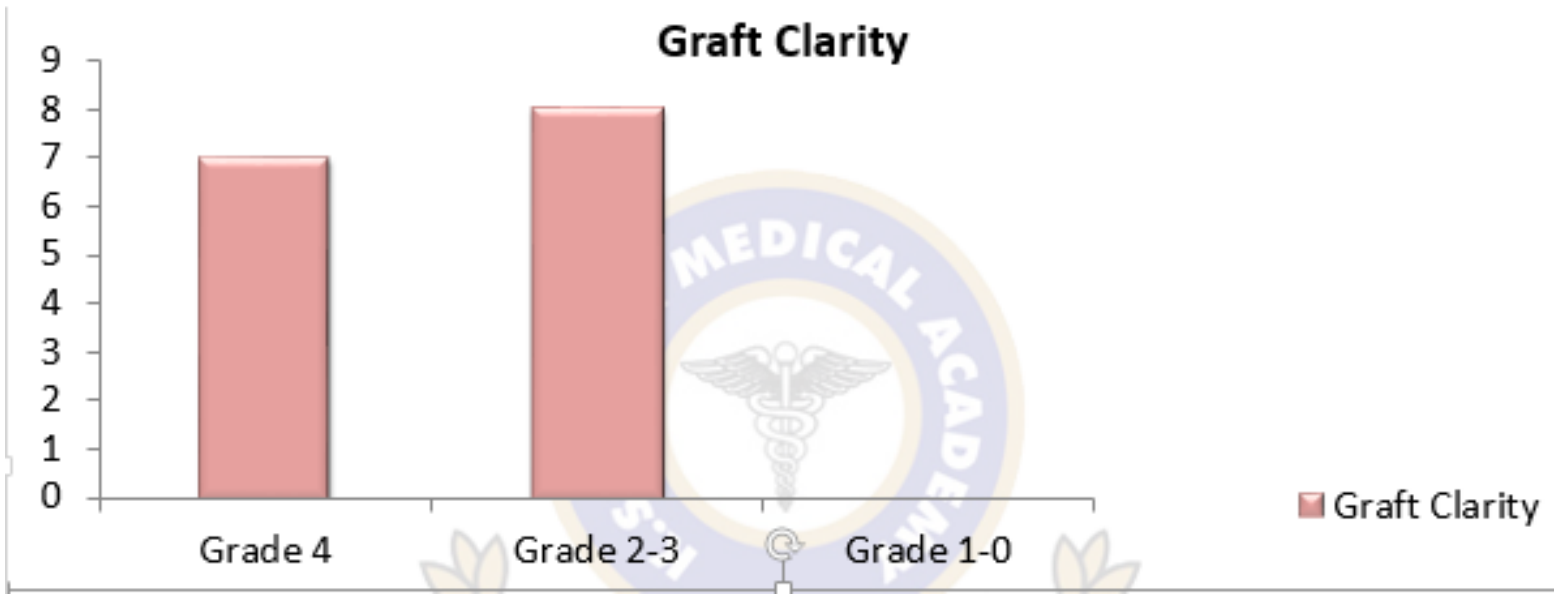

Figure 6: Post-operative graft clarity at the end of 3 months.

Table 2: Grading of Graft clarity.

\begin{tabular}{|l|l|}
\hline $\begin{array}{l}\text { Graft } \\
\text { Clarity }\end{array}$ & Impression \\
\hline Grade 4 & Optically clear with excellent view of iris details. \\
\hline Grades 2-3 & $\begin{array}{l}\text { Mid to corneal significant corneal haze with or without good view } \\
\text { of iris details. }\end{array}$ \\
\hline Grade 0 & Opaque grafts with poor view of iris and anterior segment details. \\
\hline
\end{tabular}

\section{Discussion}

Secondary IOL implantation in the absence of adequate posterior capsular support is a challenge. The modern ACIOLs with flexible haptics, though technically easier to place and easily available, can lead to significant complications including angle trauma with hyphaema, clefts, secondary glaucoma, uveitis and corneal endothelial decompansation from progressive endothelial trauma. This is important in case of a corneal graft where corneal endothelium is already compromised and there is a risk of graft rejection. In a study conducted by Marques et al. [8] ACIOL related inflammation has been the main indication for IOL exchange in more than $50 \%$ of cases. In our study, at the end of 3 months, visual acuity improvement was seen in $11(74 \%)$ eyes and remained unchanged in $4(26 \%)$ eyes. This was in accordance with the study done by Rufer et al. [9]. However combined surgery limited the refractive results with the unpredictable mire changes, which are likely postoperatively. The causes of poor vision were cystoid macular odema and macular scar noted in 2 cases each. Elevated IOP was observed in 8 cases. Similarly, raised IOP was found in 1 out of 31 patients by Schallenberg et al. [10]. In another study done by Jare et al. [11] 3 out of 108 eyes had raised IOP in the first week postoperatively which were managed with $\mathrm{Nd}$ : YAG peripheral iridectomy. Intraocular lens was stable and no significant decentrations were noted. One eye presented with hyphema in the first postoperative week, which was cleared without further treatment.

\section{Conclusion}

In our study, we found that there was a definite improvement in visual acuity and a decreased complication rate in this combined procedure. Thus, retropupillary iris claw lens implantation combined with penetrating keratoplasty is a simple and safe alternative to achieve pseudophakia in eyes with corneal edema and inadequate posterior capsular support.

\section{Limitations}

Although this study showed that retropupillary Iris claw lens is better alternative, it was limited by its modest sample size. Thus, studies with larger sample size should be conducted in the future to validate our findings. Another limitation was shorter duration in post-operative evaluation; atleast 1 year of assessment would be required to validate the study. Comparison study can also be done with regard to scleral fixation lens, as it is another alternative to treat aphakia.

\section{Ethical Clearance}

An Informed Consent was taken from all the participants. The Ethical Clearance was obtained from Institutional Ethics Committee, K. S. Hegde Medical Academy.

\section{References}

1. Worst JG. Iris claw lens. J Am Intraocul Implant Soc 1980;6:166-7.

2. Menezo JL, Cisneros AL, Rodriguez-Salvodar V. Endothelial study of iris claw phakic lens: Four year follow-up. J Cataract Refract Surg. 1998;24:1039-49. 
3. Brasse K, Neuhann TH. Posterior chamber verisyse lens implantation to correct aphakia without capsular support. Video J Cataract Refract Surg. 2004;20.

4. Rijneveld WJ, Beekhuis WH, Hassman EF, et al. Iris claw lens: Anterior and posterior iris surface fixation in the absence of capsular support during penetrating keratoplasty. J Refract Corneal Surg. 1994;10:14-9.

5. Rüfer F, Saeger M, Nölle B, et al. Implantation of retropupillar iris claw lenses with and without combined penetrating keratoplasty. Graefes Arch Clin Exp Ophthalmol. 2009;247:457-62.

6. Kanellopoulos AJ. Penetrating keratoplasty and artisan iris-fixated intraocular lens implantation in the management of aphakic bullous keratopathy. Cornea. 2004;23:220-4.

7. Shanbhag N, Cholera PP, Sahana N, et al. Evaluation of graft clarity post-penetrating keratoplasty. Int J Sci Study. 2017;5:90-6.

8. Faria M, Pinto FN, Medeiros PJ, et al. Retropupillary iris claw intraocular lens implantation in aphakia for dislocated intraocular lens (Corrigendum). Int Med Case Rep J. 2016;9:261-5.
9. Rüfer F, Saeger M, Nölle B, et al. Implantation of retropupillar iris claw lenses with and without combined penetrating keratoplasty. Graefes Arch Clin Exp Ophthalmol. 2008;247:457-62.

10. Schallenberg M, Dekowski D, Hahn A, et al. Aphakia correction with retropupillary fixated iris-claw lens (Artisan) long-term results. Clin Ophthalmol. 2014;8:137-41.

11. Jare NM, Kesari AG, Gadkari SS, et al. The posterior irisclaw lens outcome study: 6-month follow-up. Indian J Ophthalmol 2016;64:878-8.

\section{Correspondence to:}

Reshma Raj

KS Hegde Medical Academy

Thaliparamba, Kannur

Kerala, India

E-mail: dr.reshraj@gmail.com

Tel: 00919995622881 